\title{
Horizontal coordinates transformation and residuals modelling on the territory of the Republic of Srpska
}

\author{
Slavko Vasiljevićc ${ }^{*}$, Dejan Vasić ${ }^{1}$, Oleg Odalović ${ }^{2}$, Dragan Blagojević \\ Branko Milovanović ${ }^{2}$
}

1. Faculty of Architecture, Civil Engineering and Geodesy, University of Banja Luka, Banja Luka, Republic of Srpska, Bosnia and Herzegovina,

2. Faculty of Civil Engineering, University of Belgrade, Belgrade, Serbia

*Corresponding author, email: slavko.vasiljevic@aggf.unibl.org

Slavko Vasiljević - Senior Teaching Assistant, Department of geodesy, scientific field: geodetic surveying.

Dejan Vasić - Senior Teaching Assistant, Department of geodesy, scientific field: geodetic reference systems. (https://orcid.org/0000-0001-7676-0573)

Oleg Odalović - Associate Professor, Department of geodesy and geoinformatics, scientific fields: geodetic reference frames and gravity field determination. (https://orcid.org/0000-0001$\underline{6004-824 X}$ )

Dragan Blagojević - Full Professor, Department of geodesy and geoinformatics, scientific field: geodetic reference frames.

Branko Milovanović - Associate Professor, Department of geodesy and geoinformatics, scientific field: engineering geodesy. 


\title{
Horizontal coordinates transformation and residuals modelling on the territory of the Republic of Srpska
}

\begin{abstract}
The paper describes the optimal model for coordinate transformation between ETRS89 and old national reference system in the Republic of Srpska. The Helmert's parameters were estimated, based on a set of 1741 common points. The average magnitude of residuals was $0.3 \mathrm{~m}$ per coordinate and the residual vectors were homogeneous at the local level. The basic model was complemented by a corrections grid that uses bilinear interpolation. The accuracy of the transformed horizontal positions obtained by this model is about $10 \mathrm{~cm}$. In this way, a link between the ETRS 89 and local reference system has been established on the overall territory of the Republic of Srpska.
\end{abstract}

Keywords: transformation of coordinates, Helmert transformation, ETRS89, national triangulation network, grid of residuals

\section{Introduction}

Geodetic reference systems based on Global Navigation Satellite Systems (GNSS) technology are geocentric and three-dimensional. Their modern-day realisation is predominantly carried out in the form of the Continuously Operating Reference Station (CORS) networks (Jekeli and Montenbruck 2017). Before the GNSS era, national geodetic reference frames were established using terrestrial measurement methods. These traditional geodetic datums used local ellipsoids with topocentric positioning. Consequently, the origins of these systems are only approximately aligned with the Earth's centre of mass, and neither is the orientation of their axes in full accordance with the adopted conventions.

It is not uncommon for old and new geodetic reference systems to simultaneously exist and be in use in some countries. The reason is that data from a national survey, Cadastre and other public institutions have been collected over a long period of time and are mostly related to the old reference systems. CORS networks, on 
the other hand, provide quick and easy positioning within the global reference system. In order to fully utilise the GNSS technology and, at the same time, for the results to be compatible with existing data, a compromise was found in the form of a datum transformation between systems (Hofmann - Wellenhof et al. 2008).

The old national reference system in the Republic of Srpska (RS), which has been in use for more than 80 years, is based on the local Bessel ellipsoid and the Transverse Mercator projection. It is generally referred to as National Coordinate System (Državni koordinatni sistem - DKS). The system's realisation was made through the former Yugoslav's triangulation network of several orders. Coordinates were determined over a long period of time using various measurement technologies and methodologies. In order to achieve modern geodetic standards and to create the basis for the application of new measurement technologies, the Network of Continuously Operating Reference GNSS Stations of the Republic of Srpska (SRPOS) has been used in the RS since 2011. This network represents the primary realisation of a newly adopted spatial reference system, the European Terrestrial Reference System 1989 (ETRS89). The system is a Cartesian, three-dimensional geocentric system fixed to the stable part of the Eurasian plate (Altamimi 2018). In principle, point positions in this system are expressed by Cartesian coordinates $(X, Y, Z)$, while the ellipsoid GRS80 is used for the geodetic coordinates $(B, L, h)$. Universal Transverse Mercator (UTM) projection and coordinate system is used to describe positions in the horizontal plane.

In recent years, the Republic Administration for Geodetic and Property Affairs of the Republic of Srpska (Republička Uprava za Geodetske i Imovinsko-pravne Poslove - RUGIP), in cooperation with the World Bank and Swedish Lantmäteriet (the Swedish mapping, cadastral and land registration authority), has been carrying out the re-establishment of national survey, the cadastral system modernisation and the 
development of National Spatial Data Infrastructure (NSDI) (RUGIP 2020b). These tasks require rapid and accurate acquisition of spatial data, especially data related to parcels and buildings. The acquisition requires both surveying and demarcation of these physical features, which are shown on the maps, whose coordinates are realised in the legacy DKS coordinate system. The primary surveying tool here is GNSS technology referencing back to the SRPOS network, as it significantly reduces time required to execute these technical activities. However, the coordinates from GNSS surveying / SRPOS are in the ETRS89. The aforementioned projects, the increasing number of users of SRPOS network, the constant need for data integration and interoperability, as well as the capacity building for the transition to the new reference system, have imposed the need to define a suitable transformation model between ETRS89 and DKS.

The paper shows the determination of parameters of an optimal datum transformation model for the RS. A Helmert transformation was applied to the entire RS region, followed by residuals grid corrections. The Helmert transformation parameters make a general tie between DKS and ETRS89, and the grid of residuals removes the majority of the horizontal coordinate deformations.

\section{National triangulation network in the Republic of Srpska}

Properties of the modern-day national triangulation network (NTN) in the RS are conditioned by the historical legacy of the triangulation network of the former Yugoslavia. The triangulation network has been developed by several institutions over a period of almost 100 years (Odalović et al. 2016):

- Military Geography Institute of the former Austrian and Austro-Hungarian empire, in Vienna (Militaergeographisches Institut, Wien - MGI), 
- Military Geographical Institute of the former Kingdom of Yugoslavia and Socialist Federal Republic of Yugoslavia, in Belgrade (Vojnogeografski institut, Beograd - VGI),

- Federal Geodetic Administration of the former Socialist Federal Republic of Yugoslavia, in Belgrade (Savezna geodetska uprava, Beograd - SGU).

The development of the first-order triangulation network was started in 1872 by the Viennese MGI. It was a part of the Austro-Hungarian first-order triangulation. Two chains of triangles were established - from Slovenia to Vojvodina, and from Slovenia via Dalmatia to Montenegro. The latter covers the southern part of the RS, along the border with Montenegro. The two chains are connected by a third chain of triangles, that encompasses the eastern part of the RS. Geodetic datum of this triangulation was a Bessel's ellipsoid. Its position was defined by astronomical coordinates of the origin point in Hermannskogel near Vienna, and the orientation was given by the astronomical azimuth of the Hermannskogel-Hundsheimer Berg line.

Between the two world wars, the VGI of the Kingdom of Yugoslavia made efforts to improve and complete first order triangulation. The northeastern part of the RS is covered with network developed during this period. At the same time, a first-order triangulation network was established in the western part of the RS, and the work was carried out by the Cadastre department of the Ministry of Finance, with the later assistance of the VGI (Božić and Blagojević 2008).

After the World War II, VGI and SGU were working to cover the entire state territory by first-order triangulation, as well as to fill it up with lower-order networks. A first-order network that was established, had an average distance between points of 30 $\mathrm{km}$. This network was never adjusted as a whole, and the coordinates of the Viennese MGI points were used for calculations. It was soon discovered that this network did not 
meet the accuracy criteria prescribed by the International Association of Geodesy (IAG), and the design and implementation of a new, astro-geodetic network began. In the late 1960s, the planned measurements in this network were completed. However, in 1974, just at the time of the data processing, the SGU was abolished and the activities were suspended. As the astro-geodetic network wasn't completed, the coordinates of triangulation stations in the RS date from the period prior to the World War II.

The network remaining in use was divided into four orders, with distances between fourth-order points from $1 \mathrm{~km}$ to $4 \mathrm{~km}$ (Figure 1). The main disadvantages of this network are that it is heterogeneous and has low accuracy. Heterogeneity is present due to the fact that the network was never measured and adjusted as a whole, but consists of several parts that have been developed at different epochs. The low accuracy is caused by limitations in the then available measurement technologies. Subsequent studies showed that the accuracy of absolute positions of the first-order triangulation stations is about $0.5 \mathrm{~m}$ (Blagojević 2009). The relative accuracy of lower-order points is better, but it does not contain errors of the first-order points positions. In addition, the effects of geodynamic phenomena described in detail in (Vasić et al. 2018), over a long period of time (about 150 years) resulted in network distortions. Considering the present-day achievements in geodetic methodology and in the technology of measuring instruments, it can be said that the old national horizontal reference system of the RS no longer meets the modern requirements of accuracy and reliability, either by its definition or realisation.

\section{Realisations of ETRS89 in the Republic of Srpska}

ETRS89 was chosen as the new national three-dimensional (3D) reference system of the RS, with the horizontal system defined as its subset (RUGIP 2004). Its realisation, in the form of a relatively homogeneous field of points physically materialised throughout the 
territory, took place in several stages.

In September 1998, a EUREF GPS measurement campaign was conducted in the RS, as part of the BALKAN98 campaign (Altiner et al. 1999, Blagojević 2009). On this occasion, measurements at 5 first-order triangulation stations were made. The positional accuracy of the geodetic coordinates was estimated at $3 \mathrm{~mm}$, and the geodetic heights accuracy at about $7 \mathrm{~mm}$. In 2000, the density of the network was increased by additional measurements at another 10 first- and second-order triangulation points.

In the period from 2007 to 2009 , the RS made a realisation of the national spatial reference system in the form of a national reference network (Passive GPS Reference Network of the RS). The network has 327 points, including those from BALKAN98 campaign. The points are uniformly distributed, with an average distance of $10 \mathrm{~km}$. Their positional accuracy is $2 \mathrm{~cm}$ (Blagojević 2009). In larger urban areas, this network includes points of the local 3D reference networks. Some points of these networks were used to validate the proposed transformation model.

As mentioned above, since 2011, the SRPOS network has been used in the RS as the primary realisation of the new spatial reference system. The network today is comprised of 21 continuously operational GNSS reference stations, at an average distance of $50 \mathrm{~km}$ to $70 \mathrm{~km}$. They are under the jurisdiction of the Republic Administration for Geodetic and Property Affairs (Republička Uprava za Geodetske i Imovinsko-pravne Poslove - RUGIP). The network was established as a part of the Bosnia and Herzegovina Permanent Stations Network project - BiHPOS. The second component is the network of the Federation of Bosnia and Herzegovina - FBiHPOS. In order to provide adequate geometry for both networks, mutual exchange of data is performed, so that SRPOS uses data of additional eight FBiHPOS stations (RUGIP 2020a). Also, in order to link the BiHPOS network with the EUREF Permanent GNSS 
Network (EPN), as well as for to improve the coverage in the border areas, data exchange with permanent station networks of Croatia, Serbia and Montenegro is carried out. Currently available services for positioning are shown in Table 1 . The final coordinates of permanent stations refer to ETRF2000, as officially addopted reference frame in the RS. They were estimated using 16-days measurements conducted during April 2011 (RUGIP 2020a). Permanent stations spatial distribution is given in Figure 2.

\section{Mathematical Models of Coordinate Transformation}

Models of coordinate transformations are linear and nonlinear models that create a functional relationship between coordinates in two reference systems. Their quality is defined by different parameters, on which the choice of the appropriate model depends. The seven-parameter transformation (also called Helmert transformation) in rectangular coordinates, was chosen as the optimal model for global coordinate transformation in the RS (Blagojević, 2009).

\section{Mathematical model of Helmert transformation in rectangular coordinates}

A seven-parameter coordinates transformation of an arbitrary point $P(X, Y, Z)$, from one spatial Cartesian system to another, is given by (Deakin 2006):

$$
\left[\begin{array}{c}
X_{2} \\
Y_{2} \\
Z_{2}
\end{array}\right]=\left[\begin{array}{c}
t_{x} \\
t_{y} \\
t_{z}
\end{array}\right]+(1+d m)\left[\begin{array}{ccc}
1 & \varepsilon_{z} & -\varepsilon_{y} \\
-\varepsilon_{z} & 1 & \varepsilon_{x} \\
\varepsilon_{y} & -\varepsilon_{x} & 1
\end{array}\right]\left[\begin{array}{c}
X_{1} \\
Y_{1} \\
Z_{1}
\end{array}\right]
$$

wherein:

$X_{2}, Y_{2}, \mathrm{Z}_{2}-\quad$ coordinates in the second system,

$t_{X}, t_{Y}, t_{Z}-$ translation parameters, covering up differences in positions of the two system origins, 
$1+d m-\quad$ a scale factor, because scale of linear units can differ in two systems, $\varepsilon_{X}, \varepsilon_{Y}, \varepsilon_{Z}-$ three rotation parameters (Euler angles), covering up the differences in orientation of the coordinate axes of the two systems,

$X_{1}, Y_{1}, \mathrm{Z}_{1}-\quad$ coordinates in the first system.

Equation (1) represents a functional part of the Helmert transformation mathematical model, because it relates the coordinates in the two systems to unknown transformation parameters. This model is known in the literature as the Bursa-Wolf model. For small-scale networks, the Molodensky-Badekas model is generally used, which introduces the centroid of a set of common points (Mitsakaki et al. 2006). In this manner, the high correlation of the estimated transformation parameters is avoided, and numerically more stable system is obtained. The equation is:

$$
\left[\begin{array}{c}
X_{2} \\
Y_{2} \\
Z_{2}
\end{array}\right]=\left[\begin{array}{c}
t_{x} \\
t_{y} \\
t_{z}
\end{array}\right]+\left[\begin{array}{c}
X_{C} \\
Y_{C} \\
Z_{C}
\end{array}\right]+(1+d m)\left[\begin{array}{ccc}
1 & \varepsilon_{z} & -\varepsilon_{y} \\
-\varepsilon_{z} & 1 & \varepsilon_{x} \\
\varepsilon_{y} & -\varepsilon_{x} & 1
\end{array}\right]\left[\begin{array}{c}
X_{1}-X_{C} \\
Y_{1}-Y_{C} \\
Z_{1}-Z_{C}
\end{array}\right],
$$

where $X_{C}, Y_{C}$ and $Z_{C}$ stand for centroid coordinates.

A common way for the formulation of stochastic model when estimating transformation parameters based on Cartesian coordinates, is to assume the same accuracy of measurements along all three axes, or to use standard deviations estimated from the measurement vectors processing.

\section{Interpolation Models of Transformed Coordinate Residuals}

Sometimes Helmert transformation parameters cannot provide a sufficiently accurate transformation of coordinates. Corrections of horizontal coordinates at common points, which are determined after the transformation parameters estimation, are a reliable indicator of the transformation quality. 
Horizontal coordinates $y_{T}$ and $x_{T}$, calculated using Helmert transformation, can easily be reduced to their original values $y_{2}$ and $x_{2}$ :

$$
\begin{aligned}
& y_{2}=y_{T}-v_{y}, \\
& x_{2}=x_{T}-v_{x} .
\end{aligned}
$$

However, in order for the identical procedure to be repeated at arbitrary points, it is necessary to predict the corrections $v_{y}$ and $v_{x}$ in these positions. Although these can be derived directly from residuals at common points, using some type of interpolation procedure, the practical and commonly used method includes a rectangular grid of residuals. Such grid-based transformation procedures have already been adopted in many countries, such as Australia (Collier 2002; Kinneen, et al. 2004), Austria (Imrek 2005), Germany (Kempe et al. 2006), United Kingdom (Ordnance Survey 2013), Netherlands (Broekman et al. 2013), Serbia (Odalović et al. 2016), Croatia (Grgić et al. 2016), etc.

Residuals in grid nodes can be calculated using the values at common points located in nodes proximity (Figure 3), according to the principle of weighted mean:

$$
\begin{aligned}
& v_{y_{P}}=\frac{1}{\sum_{i=1}^{n} p_{i}} \sum_{i=1}^{n} p_{i} v_{y_{i}}, \\
& v_{x_{P}}=\frac{1}{\sum_{i=1}^{n} p_{i}} \sum_{i=1}^{n} p_{i} v_{x_{i}} .
\end{aligned}
$$

\section{In Figure 3, $R$ stands for radius of area inside of which common points were} used. The weights are calculated as the powers of reciprocal distances from the grid node to the common points, according to the formula (Lu and Wong, 2008): 


$$
p_{i}=\frac{1}{d_{P i}^{q}}=\frac{1}{\left(\sqrt{\Delta y_{P i}^{2}+\Delta x_{P i}^{2}}\right)^{q}},
$$

where $q$ is the power of reciprocal distance $d_{P i}$.

This approach gives an estimation of corrections in predetermined locations with regular spatial distribution. To evaluate corrections at an arbitrary location, the most commonly applied procedure is bilinear interpolation (Collier 2002; Greaves 2004), the basic principle of which is illustrated in Figure 4.

For the location $(y, x)$ in left-handed coordinate system DKS, in which the prediction is to be done, the local normalised coordinates $y_{L}$ and $x_{L}$ are calculated. They refer to the southwestern node of the grid $\left(y_{0}, x_{0}\right)$ :

$$
\begin{aligned}
& y_{L}=\frac{y-y_{0}}{d y}, \\
& x_{L}=\frac{x-x_{0}}{d x},
\end{aligned}
$$

wherein $d_{Y}$ and $d_{X}$ are grid steps along the coordinate axes (lengths of grid cell sides). If $f(0,0) ; f(1,0) ; f(0,1) ; f(1,1)$ denote known residual values in four nodes of the grid cell, and $f(y, x)$ denotes predicted residual values, then the bilinear interpolation is carried out using the formula:

$$
\begin{aligned}
f(y, x)= & f(0,0)\left(1-x_{\mathrm{L}}\right)\left(1-y_{\mathrm{L}}\right)+f(1,0) x_{\mathrm{L}}\left(1-y_{\mathrm{L}}\right)+ \\
& +f(0,1)\left(1-x_{\mathrm{L}}\right) y_{\mathrm{L}}+f(1,1) x_{\mathrm{L}} y_{\mathrm{L}} .
\end{aligned}
$$

\section{Determination of Transformation Parameters and Residuals Grid in the Republic of Srpska}

Horizontal transformation of coordinates on the territory of the RS, between the two 
aforementioned systems, is carried out in two steps: global seven-parameter transformation and additional position corrections using a grid of residuals. Seven parameters and the grid were determined using a set of common points, with known positions both in the old reference system (DKS) and in ETRS89.

\section{Set of common points in two reference system}

The basic data set for determination of the transformation parameters and the residuals grid consisted of 1758 points, with coordinates known in both systems (Figure 5). These are triangulation points of all orders, with ellipsoidal heights determined using trigonometric levelling (Odalović and Grekulović 2013). Their ETRS89 coordinates were determined using the SRPOS network and GPSP service. It is obvious that points distribution on the territory of the RS is homogeneous, and as such they can be used to estimate transformation parameters.

\section{Statistical analysis of data sets}

Statistical analyses were made using preliminary estimated transformation parameters. The obtained coordinates corrections represent a measure of agreement between the old and the new system. Based on $n$ corrections $v_{y}$ and $v_{x}$, the total position deviation per each point is calculated:

$$
v_{P_{i}}=\sqrt{v_{y_{i}}^{2}+v_{x_{i}}^{2}} .
$$

These deviations of each common point are compared to the standard deviation of horizontal position in the entire network, calculated as: 


$$
\sigma_{y}=\sqrt{\frac{\sum_{i=1}^{n} v_{y_{i}}^{2}}{n}}, \sigma_{x}=\sqrt{\frac{\sum_{i=1}^{n} v_{x_{i}}^{2}}{n}}, \sigma_{P}=\sqrt{\sigma_{y}^{2}+\sigma_{x}^{2}} .
$$

In order to keep a point in the set, a condition had to be met:

$$
v_{P_{i}} \leq 3 \sigma_{P}, \quad i=1,2, \ldots, n
$$

Otherwise the point would be excluded from the set, and the whole process would be repeated. Only one point is excluded at each step. According to the described procedure, a total of 17 points were excluded, reducing the initial set to 1741 common points.

The accuracy of the points horizontal positions in the ETRS89 system is about $1 \mathrm{~cm}-2 \mathrm{~cm}$. Given that the coordinates are determined for each point individually, they can be considered as uncorrelated. Due to the high accuracy of the ETRS 89 coordinates, they were further considered as true. The accuracy of the horizontal positions in DKS was estimated from coordinates corrections, obtained using preliminary estimated transformation parameters. The horizontal position standard deviations were calculated according to equations (9), and the following values were obtained: $\sigma_{y}=0.30 \mathrm{~m}$, $\sigma_{x}=0.33 \mathrm{~m}$ and $\sigma_{P}=0.45 \mathrm{~m}$.

However, the values calculated in this way express average quality. They refer to the network centroid, and depend on the size of the area where common points are placed. Since the relative positional accuracy of the triangulation network points depends on the distance between them, the numerical value of this dependence was determined as a better indicator of quality.

Deviation differences between pairs of points were calculated in all combinations, according to the formulas: 


$$
\begin{aligned}
& v_{y_{i j}}=v_{y_{j}}-v_{y_{i}}, \\
& v_{x_{i j}}=v_{x_{j}}-v_{x_{i}} .
\end{aligned}
$$

The calculated deviation differences are grouped according to the distance between points, with characteristic distances of $5 \mathrm{~km}$ being adopted. The standard deviations of the relative horizontal positions were then calculated for each characteristic distance:

$$
\sigma_{d y}=\sqrt{\frac{\sum_{k=1}^{n_{c}} v_{y_{j, k}}^{2}}{n_{c}}}, \sigma_{d x}=\sqrt{\frac{\sum_{k=1}^{n_{c}} v_{x_{i j, k}}^{2}}{n_{c}}},
$$

with $n_{c}$ standing for a number of combinations. Figure 6 shows the total dispersions as a function of the distances between triangulation points, and the regression line calculated using the method of the least squares - MLS (Ghilani 2010).

It can be seen that straight line fits the dispersions of relative positions very well, and their values increase with increasing distance. The regression coefficient (slope) of the regression line is $0.00352 \mathrm{~m}^{2} / \mathrm{km}$. It follows the quality of the relative horizontal positions of the triangulation points which can be expressed by the standard deviation $\sigma_{d_{P}}=0.059 \mathrm{~m} / \sqrt{\mathrm{km}}$. Accordingly, the coordinates of triangulation points are not mutually independent. The level of correlation between adjacent stations is a function of the distance between them, and arises from identical measuring procedures and methods of coordinates calculation.

The basis for calculation of coordinates stochastic dependence, are the deviations along the coordinate axes at common points. The first step is to calculate the product of the deviations between pairs of points in all combinations, according to formulas: 


$$
\begin{aligned}
& v_{y_{i j}}=v_{y_{i}} \cdot v_{y_{j}}, \\
& v_{x_{i j}}=v_{x_{i}} \cdot v_{x_{j}} .
\end{aligned}
$$

These products are then classified according to the distances between points, with characteristic values of $5 \mathrm{~km}$. For each distance, the discrete covariances of horizontal positions are calculated using formulas:

$$
c_{y}=\frac{\sum_{k=1}^{n_{c}} v_{y_{i j, k}}}{n_{c}}, c_{x}=\frac{\sum_{k=1}^{n_{c}} v_{x_{i j, k}}}{n_{c}} .
$$

Correlation coefficients are calculated as:

$$
r_{y}=\frac{c_{y}}{\sigma_{y}^{2}}, r_{x}=\frac{c_{x}}{\sigma_{x}^{2}}
$$

Figure 7 shows the correlation coefficients as a function of the distances between points, and the discrete autocorrelation function. It can be seen that they are almost identical for both coordinate axes, and that the coordinates have high correlation up to the distances of about $20 \mathrm{~km}$, at which the correlation decreases to a value of about 0.4 . For lengths from $60 \mathrm{~km}$ to $100 \mathrm{~km}$, the coordinates can be considered practically independent, since the correlation coefficient is close to zero.

\section{Estimated transformation parameters}

Based on a set of common points, the parameters of general Helmert transformation (Table 2) were calculated for the entire territory of the RS. The Molodensky-Badekas model was adopted, and system of equations (2) was solved using MLS. Regarding stochastic model, same accuracy along all three axes was assumed. The flowchart of parameters determination is given in Figure 8. 
Based on these parameters, the transformed coordinates of the common points are calculated. Using formulas (3), the horizontal coordinates residuals are obtained. Information on residuals is given in Table 3, and their graphical representation is given in Figure 9. The figure shows that the residuals are homogeneous at the local level (several tens of $\mathrm{km}$ ), which is expected given the results of the statistical analysis, that showed a high correlation of coordinates up to a distance of $20 \mathrm{~km}$. It is this fact that allows successful application of the residual grid.

\section{Grid of residuals}

Helmert transformation gives positional deviations at common points of about $0.5 \mathrm{~m}$. Therefore, a grid of residuals is introduced so that for a point at an arbitrary location, the transformed horizontal position can be determined with better accuracy at the local level.

The simplest use of residuals, that is, their interpolation for arbitrary locations, is when they are in the form of a grid. A grid of residuals covers the entire territory of the $\mathrm{RS}$ and has a resolution of $1 \mathrm{~km}$. In this way, the territory of the RS is covered by a grid with 303 rows and 273 columns. Other grid parameters are shown in Table 4.

Entire procedure of coordinates transformation of an arbitrary point, using both general transformation parameters and grid of residuals, is shown in Figure 10. The same procedure was used to predict corrections at common points.

The accuracy of residuals grid was tested using differences between known and predicted residuals at common points. Figure 11 shows the histograms of residuals differences. It can be seen that their distribution closely matches normal distribution. Table 5 contains basic statistical information of the differences.

The extreme values shown in Table 5 are exceptional cases that remain after the procedure for gross error testing is finished, according to the previously described 
methodology. It is obvious that the differences are centered (mean is $0.00 \mathrm{~m}$ ) and have a small standard deviation (only $0.04 \mathrm{~m}$ ).

The histogram of the total horizontal position differences in meters (calculated from differences in coordinates) is shown in Figure 12. These differences have a distribution that can be approximated by chi-squared distribution. The standard deviation of the position is $0.056 \mathrm{~m}$.

Finally, Figure 13 shows the improvement in the quality of horizontal position transformation, by comparing the residuals after the Helmert transformation only, and after the interpolation with the residuals grid.

Accuracy of the residual grid was tested using 200 points whose coordinates are known in ETRS89 and DKS, and whose distribution over the territory of the RS is homogeneous. These are mainly points of local spatial networks, that were not included in transformation parameters and residuals grid calculations. Their DKS coordinates were predicted using transformation parameters and residuals grid interpolation. The histogram of the known and predicted horizontal positions differences closely matches the chi-squared distribution (Figure 14). The standard deviation of the horizontal position is $0.106 \mathrm{~m}$.

\section{Discussion and Conclusion}

A seven-parameter transformation, improved by a grid of residuals, is an optimal model of horizontal transformation in the territory of the RS. The accuracy of the horizontal position obtained from this model is about $10 \mathrm{~cm}$, which satisfies the accuracy requirements in the majority of practical works, according to legal regulations. This solves the problem of connecting the old and new reference system in the entire territory of the RS. 
The adopted model has the following properties: allows for two-way general transformation, is relatively simple, is unique, covers deformations, refers to horizontal positions of points and has adequate accuracy. The transformation model enables national institutions to make strategic, normative and operational decisions during the transitional period of coexistence of the old and new reference system. It allows positioning for the purposes of national survey, Cadastre, engineering, GIS applications, etc. Additional aspects are the harmonisation of the national reference system with the European reference system and systems of neighboring countries, participation in European projects and regional geodetic cooperation, efficient and reliable land, air and river navigation throughout the RS, and to some extent beyond its borders.

The transformation model will meet many practical needs related to:

- support for geodetic and cadastral automatisation,

- support for the growing needs of the state administration,

- more efficient and easier management of spatial data,

- reduction of costs and risks of the state administration,

- the possibility of wider use of modern geodetic measuring systems, primarily GNSS,

- compatibility and interoperability with international systems, etc.

The application of the GNSS technology will significantly simplify activities on national survey and spatial data acquisition in general, including parcel and building demarcation. This will, indirectly, improve the whole procedure of the NSDI development, in accordance to the INSPIRE (INfrastructure for SPatial InfoRmation in the European Community) directive, and add to the modernisation of the RS cadastral system. Accurate and reliable geospatial data in the RS create a basis for: optimal real 
estate management, efficient real estate market, tax system, national statistical system, mortgage banking development, investments, economic growth and support to the increasing needs of the state administration.

The use of adopted model is still limited in urban areas. Considering the high prices of real estate, the expected position accuracy of $10 \mathrm{~cm}$ is often not sufficient. The problem is that points from local networks were not used in parameters estimation and in the creation of residuals grid, and the real estate data were obtained relying precisely on those networks.

In order to improve the grid of residuals and the overall accuracy of the transformation, additional data will need to be collected in the future. This includes determining the ETRS89 coordinates for as many reference networks points as possible. It is desirable to create a database of common points, which would serve to control the quality of new points. At the same time, it would be the basis for quick and easy calculation of new parameters and residuals grid, their integration into the SRPOS network software and distribution of transformed coordinates to users within network corrections. This would improve the model and raise the efficiency of its application to a higher level. 


\section{References}

Altamimi, Z., 2018. Relationship and Transformation between the International and the European Terrestrial Reference System. EUREF Technical Note 1. 28 June.

Altiner, Y., Schlueter, W., and Seeger, H., 1999. Results of the Balkan98 GNSS campaigns in Albania, Bosnia and Herzegovina, and Yugoslavia. Report on the symposium of the IAG subcommission for the European Reference Frame (EUREF), 2 - 5 June 1999, Prague, Czech Republic.

Blagojević, D., 2009. Project of horizontal transformation in the territory of the Republic of Srpska (in Serbian). Republic Administration for Geodetic and Property Affairs of the Republic of Srpska. Banja Luka. Technical Report.

Božić, B. and Blagojević, D., 2008. Strategy of establishing fundamental geodetic reference systems in the territory of the Republic of Srpska (in Serbian). Republic Administration for Geodetic and Property Affairs of the Republic of Srpska. Banja Luka. Technical Report.

Broekman, R., Marel, H. and Huisman, L., 2013. National report of the Netherlands. Symposium of the IAG Subcommission for Europe (EUREF), 29 - 31 May 2013, Budapest, Hungary.

Collier, P., 2002. Development of Australia's national GDA94 transformation grids. Consultant's report to the Intergovernmental Committee on Surveying and Mapping, Univ. of Melbourne, Australia.

Deakin, R. E., 2006. A note on the Bursa-Wolf and Molodensky-Badekas Transformations, School of Mathematical and Geospatial Sciences, RMIT University, 1-21, Tech. rep.

Ghilani, C. D., 2010. Adjustment computations. $5^{\text {th }}$ edition. Hoboken: John Wiley \& Sons.

Greaves, M., 2004. OSTN02: A New Definitive Transformation From GPS Derived Coordinates to National Grid Coordinates in Great Britain. Survey Review, 37 (293), 502-519.

Grgić, M., Varga, M. and Bašić, T., 2016. Empirical Research of Interpolation Methods in Distortion Modeling for the Coordinate Transformation between Local and Global Geodetic Datums. Journal of Surveying Engineering, 142(2). 
Hofmann-Wellenhof, B., Lichtenegger, H. and Wasle, E., 2008. GNSS-global navigation satellite systems: GPS, GLONASS, Galileo, and more. $1^{\text {st }}$ edition., New York: Springer.

Imrek, E., 2005. Homogenisation of the Austrian field of control points - preparatory work for a higher absolute accuracy in cadastre. Report on the Symp. of the IAG Subcommission for Europe (EUREF). Reports of the EUREF Technical Working Group (TWG). No. 15, p. 153. Germany: Bundesamt für Kartographie und Geodäsie, Frankfurt am Main

Jekeli, C. and Montenbruck, O., 2017. Time and reference systems. In: Peter J. G. Teunissen, Oliver Montenbruck, Eds. Springer Handbook of Global Navigation Satellite Systems. New York, Springer, 25-58.

Kempe, C., Alfredsson, A., Engberg, L. E., and Lilje, M., 2006. Correction model to rectify distorted co-ordinate systems. XXIII International FIG Congress (INTERGEO), 8 - 13 October Munich, Germany.

Kinneen, R.W. and Featherstone, W.E., 2004. Empirical Evaluation of Published Transformation Parameters from the Australian Geodetic Datums (AGD66 and AGD84) to the Geocentric Datum of Australia (GDA94). Journal of Spatial Science, 49 (2), 1-29.

Lu, G. Y., and Wong, D. W., 2008. An adaptive inverse-distance weighting spatial interpolation technique. Computers \& Geosciences. 34 (9), 1044-1055.

Mitsakaki, C., Agatza-Balodimou, A. M. and Papazissi, K., 2006. Geodetic reference frames transformations. Survey Review. 38 (301), 608-618.

Odalović, O., Grekulović, C. S., 2013. Transformation of classical geodetic control networks to International Terrestrial Reference Frame by transformation grids. 13th SGEM GeoConference on Informatics, Geoinformatics And Remote Sensing, 16 - 22 June, Albena, Bulgaria.

Odalović, O., Todorović Drakul, M., Grekulović, S., Popović, J. and Joksimović, D., 2016. Chronology of the development of geodetic reference networks in Serbia. Survey Review. 50 (359), 163-173.

Technical Report v2.2, 2013. A guide to coordinate systems in Great Britain: An introduction to mapping coordinate systems and the use of GPS datasets with ordnance survey mapping. Southampton: Ordnance Survey. 
Republic Administration for Geodetic and Property Affairs of the Republic of Srpska, 2004. Rulebook for fundamental geodetic works (in Serbian). Official Newsletter of the RS.

Republic Administration for Geodetic and Property Affairs of the Republic of Srpska, 2020a. SRPOS Network Official Website. https://www.rgurs.org/en/servisi/srpos [Accessed 7 January 2020].

Republic Administration for Geodetic and Property Affairs of the Republic of Srpska, 2020b. RERP project Official Website: http://www.rgurs.org/lat/projekatrerp/informacije [Accessed 7 January 2020].

Vasić, D. D., Todorić, Z. G., Višnjić, I. R., 2018. Geostatistical determination of recent vertical movements of the Earth's crust on the territory of the Republic of Srpska. Survey Review, 50 (362), 447-453. 
Table 1. Currently available services for positioning by SRPOS

\begin{tabular}{|c|c|c|}
\hline $\begin{array}{c}\text { Service } \\
\text { abbreviation }\end{array}$ & Service & Accuracy \\
\hline DSP & Differential real-time positioning & $1 \mathrm{~m}-3 \mathrm{~m}$ \\
\hline VPSP & High-accuracy real-time positioning & $1 \mathrm{~cm}-2 \mathrm{~cm}$ \\
\hline GPSP & High-accuracy post-processing positioning & $1 \mathrm{~cm}$ \\
\hline ACCS & Automatic Coordinates Computation Service & Depends on the \\
& & parameters \\
\hline
\end{tabular}

Table 2. Estimated parameters of Helmert transformation for the RS

\begin{tabular}{|c|c|c|}
\hline Transformation parameter & Value & Root-mean-square deviation \\
\hline$t_{X}$ & $-681.839 \mathrm{~m}$ & $0.018 \mathrm{~m}$ \\
\hline$t_{Y}$ & $203.625 \mathrm{~m}$ & $0.018 \mathrm{~m}$ \\
\hline$t_{Z}$ & $-478.638 \mathrm{~m}$ & $0.018 \mathrm{~m}$ \\
\hline$\varepsilon_{X}$ & $5.57426^{\prime \prime}$ & $0.044933^{\prime \prime}$ \\
\hline$\varepsilon_{Y}$ & $3.17599^{\prime \prime}$ & $0.05476^{\prime \prime}$ \\
\hline$\varepsilon_{Z}$ & $-12.12814^{\prime \prime}$ & $0.05056^{\prime \prime}$ \\
\hline$d_{m}$ & $-1.9322 \mathrm{ppm}$ & $0.1745 \mathrm{ppm}$ \\
\hline $\mathrm{X}_{\mathrm{C}}$ & $4353067.978 \mathrm{~m}$ & $/$ \\
\hline $\mathrm{Y}_{\mathrm{C}}$ & $1427750.687 \mathrm{~m}$ & \\
\hline $\mathrm{Z}_{\mathrm{C}}$ & $4422582.645 \mathrm{~m}$ & $/$ \\
\hline & & \\
\hline & & \\
\hline
\end{tabular}


Table 3. Elementary statistical information on residuals

\begin{tabular}{|c|c|c|}
\hline Value & $\boldsymbol{y}$ axis [m] & $\boldsymbol{x}$ axis [m] \\
\hline Max & +1.45 & -1.11 \\
\hline Min & -1.10 & 0.00 \\
\hline Average & 0.00 & 0.33 \\
\hline Standard deviation & 0.30 & \\
\hline
\end{tabular}

Table 4. Information on grid parameters

\begin{tabular}{|c|c|}
\hline Parameter & Value \\
\hline Grid latitude borders (Transverse Mercator projection) & $4712445.0 \mathrm{~m}-5015225.0 \mathrm{~m}$ \\
\hline Grid longitude borders (Transverse Mercator projection) & $6358370.0 \mathrm{~m}-6630520.0 \mathrm{~m}$ \\
\hline Resolution of residuals & $1 \mathrm{~cm}$ \\
\hline Method applied for grid calculation & Weighted mean \\
\hline Power of reciprocal distances (weights calculation) & 2 \\
\hline Radius of area inside of which common points were used & $10000 \mathrm{~m}$ \\
\hline
\end{tabular}

Table 5. Elementary statistical information on residuals differences

\begin{tabular}{|l|c|c|}
\hline \multicolumn{1}{|c|}{ Parameter } & $\boldsymbol{y}$ axis [m] & $\boldsymbol{x}$ axis [m] \\
\hline Max & +0.32 & $+0.35 \mathrm{~m}$ \\
\hline Min & -0.18 & $-0.14 \mathrm{~m}$ \\
\hline Average & $\mathbf{0 . 0 0}$ & $\mathbf{0 . 0 0}$ \\
\hline Standard deviation & $\mathbf{0 . 0 4}$ & $\mathbf{0 . 0 4}$ \\
\hline
\end{tabular}


Figure 1. Existing NTN in the RS

Figure 2. SRPOS network

Figure 3. Principle of grid creation by weighted mean method

Figure 4. Principle of bilinear interpolation

Figure 5. Set of common points on the territory of the RS

Figure 6. Total dispersions as a function of the distances between triangulation points

Figure 7. Correlation coefficients as a function of the distances between triangulation points

Figure 8 . Flowchart of parameters determination

Figure 9. Graphical representation of horizontal residuals in the RS

Figure 10. Flowchart of coordinate transformation using general transformation parameters and grid of residuals

Figure 11. Histograms of residual differences by $y$ axis (left) and $x$ axis (right)

Figure 12. Histogram of residual horizontal position differences

Figure 13. Improvement in the quality of horizontal position transformation

Figure 14. Histogram of the known and predicted horizontal positions differences on the points for grid accuracy testing 


\section{Acknowledgements}

We would like to express our gratitude to the Republic Administration for Geodetic and Property Affairs of the Republic of Srpska, for the data provided and assistance in the preparation of the article. 